\title{
Cesáro partial sums of certain analytic functions
}

\author{
Rabha W Ibrahim ${ }^{1}$ and Maslina Darus ${ }^{2 *}$
}

"Correspondence: maslina@ukm.my ${ }^{2}$ School of Mathematical Sciences, Faculty of Science and Technology, Universiti Kebangsaan Malaysia, Bangi, Selangor Darul Ehsan 43600, Malaysia

Full list of author information is available at the end of the article

\section{Abstract}

The aim of the present paper is to consider geometric properties such as starlikeness and convexity of the Cesáro partial sums of certain analytic functions in the open unit disk. By using the Cesáro partial sums, we improve some recent results including the radius of convexity.

AMS Subject Classification: $30 C 45$

Keywords: analytic function; univalent functions; starlike functions; unit disk; Cesáro partial sums; partial sums

\section{Introduction}

Let $U:=\{z:|z|<1\}$ be a unit disk in the complex plane $\mathbb{C}$ and let $\mathcal{H}$ denote the space of all analytic functions on $U$. Here we suppose that $\mathcal{H}$ is a topological vector space endowed with the topology of uniform convergence over compact subsets of $U$. Also, for $a \in \mathbb{C}$ and $n \in \mathbb{N}$, let $\mathcal{H}[a, n]$ be the subspace of $\mathcal{H}$ consisting of functions of the form $f(z)=$ $a+a_{n} z^{n}+a_{n+1} z^{n+1}+\cdots$. Further, let $\mathcal{A}:=\left\{f \in \mathcal{H}: f(0)=f^{\prime}(0)-1=0\right\}$ and $\mathcal{S}$ denote the class of univalent functions in $\mathcal{A}$. A function $f \in \mathcal{A}$ is called starlike if $f(U)$ is a starlike domain with respect to the origin, and the class of univalent starlike functions is denoted by $S^{*}$. It is called convex $\mathcal{C}$, if $f(U)$ is a convex domain. Each univalent starlike function $f$ is characterized by the analytic condition

$$
\Re\left(\frac{z f^{\prime}(z)}{f(z)}\right)>0, \quad z \in U
$$

Also, it is known that $z f^{\prime}(z)$ is starlike if and only if $f$ is convex, which is characterized by the analytic condition

$$
\Re\left(1+\frac{z f^{\prime \prime}(z)}{f^{\prime}(z)}\right)>0, \quad z \in U
$$

For a function $f(z) \in \mathcal{A}$, we introduce the partial sum of $f(z)$ by

$$
f_{k}(z)=z+\sum_{n=2}^{k} a_{n} z^{n}, \quad z \in U .
$$

For the partial sums $f_{n}(z)$ of $f(z) \in S^{*}$, Szegö [1] showed that if $f(z) \in S^{*}$, then $f_{n}(z) \in S^{*}$ for $|z|<\frac{1}{4}$ and $f_{n}(z) \in \mathcal{C}$ for $|z|<\frac{1}{8}$. Owa [2] considered the starlikeness and convexity of

\section{Springer}

(c) 2013 Ibrahim and Darus; licensee Springer. This is an Open Access article distributed under the terms of the Creative Commons Attribution License (http://creativecommons.org/licenses/by/2.0), which permits unrestricted use, distribution, and reproduction in any medium, provided the original work is properly cited. 
partial sums,

$$
f_{n}(z)=z+a_{n} z^{n},
$$

of certain functions in the unit disk. Moreover, Darus and Ibrahim [3] determined the conditions under which the partial sums of functions of bounded turning are also of bounded turning.

In this paper, we consider the Cesáro partial sums, it is showed that this kind of partial sums preserve the properties of the analytic functions in the unit disk. Robertson [4] showed that if $f(z) \in \mathcal{A}$ is univalent, then also all the Cesáro sums are univalent in the unit disk. Moreover, if its ordinary partial sums (1) is univalent in $U$, then the Cesáro sums are univalent. By employing the concept of the subordination, these results were extended by Ruscheweyha and Salinas [5]. The classical Cesáro means play an important role in geometric function theory (see [6-10]).

From the partial sum

$$
s_{k}(z)=\sum_{n=1}^{k} a_{n} z^{n}, \quad z \in U,
$$

with $a_{1}=1$, we construct the Cesáro means $\sigma_{k}(z)$ of $f \in \mathcal{A}$ by

$$
\begin{aligned}
\sigma_{k}(z) & =\frac{1}{k} \sum_{n=1}^{k} s_{n}(z) \\
& =\frac{1}{k}\left[s_{1}(z)+\cdots+s_{k}(z)\right] \\
& =\frac{1}{k}\left[z+\left(z+a_{2} z^{2}\right)+\cdots+\left(z+\cdots+a_{k} z^{k}\right)\right] \\
& =\frac{1}{k}\left[k z+(k-1) a_{2} z^{2}+\cdots+a_{k} z^{k}\right] \\
& =z+\sum_{n=2}^{k}\left(\frac{k-n+1}{k}\right) a_{n} z^{n} \\
& =f(z) *\left[z+\sum_{n=2}^{k}\left(\frac{k-n+1}{k}\right) z^{n}\right] \\
& =f(z) * g_{k}(z),
\end{aligned}
$$

where

$$
g_{k}=z+\sum_{n=2}^{k}\left(\frac{k-n+1}{k}\right) z^{n} .
$$

Our aim is to consider geometric properties such as starlikeness and convexity of the Cesáro partial sums of certain analytic functions in the open unit disk.

\section{Main results}

We define the function $S_{k}$ which is a partial sum of $f \in \mathcal{A}$ by

$$
S_{k}(z)=z+\left(\frac{a_{k}}{k}\right) z^{k}, \quad k \geq 2, a_{k} \neq 0 .
$$


Theorem 1 The function $S_{k}(z)$ satisfies

$$
\frac{1-\left|a_{k}\right| r^{k-1}}{1-\frac{\left|a_{k}\right|}{k} r^{k-1}} \leq \Re\left(\frac{z S_{k}^{\prime}(z)}{S_{k}(z)}\right) \leq \frac{1+\left|a_{k}\right| r^{k-1}}{1+\frac{\left|a_{k}\right|}{k} r^{k-1}}
$$

for

$$
0 \leq r<\sqrt[k-1]{\frac{k}{\left|a_{k}\right|}} \leq 1, \quad\left|a_{k}\right| \neq 0 .
$$

Furthermore, $S_{k}(z) \in S^{*}(\alpha)$ for

$$
0 \leq r<\sqrt[k-1]{\frac{1-\alpha}{(1-\alpha / k)\left|a_{k}\right|}} \leq 1, \quad\left|a_{k}\right| \neq 0 .
$$

Proof Noting that

$$
\frac{z S_{k}^{\prime}(z)}{S_{k}(z)}=k-\frac{(k-1)}{1+\frac{a_{k}}{k} z^{k-1}}
$$

it follows that for $\cos \theta \rightarrow 1$, we obtain

$$
\begin{aligned}
\Re\left(\frac{z S_{k}^{\prime}(z)}{S_{k}(z)}\right) & =k-(k-1) \frac{1+\frac{\left|a_{k}\right|}{k} \cos \theta r^{k-1}}{1+2 \frac{\left|a_{k}\right|}{k} r^{k-1} \cos \theta+\left(\frac{\left|a_{k}\right|}{k}\right)^{2} r^{2(k-1)}} \\
& \leq \frac{1+\left|a_{k}\right| r^{k-1}}{1+\frac{\left|a_{k}\right|}{k} r^{k-1}} .
\end{aligned}
$$

Moreover, we also observe that

$$
\Re\left(\frac{z S_{k}^{\prime}(z)}{S_{k}(z)}\right) \geq \frac{1-\left|a_{k}\right| r^{k-1}}{1-\frac{\left|a_{k}\right|}{k} r^{k-1}} .
$$

Now assume that

$$
\frac{1-\left|a_{k}\right| r^{k-1}}{1-\frac{\left|a_{k}\right|}{k} r^{k-1}}>\alpha
$$

for

$$
0 \leq r<\sqrt[k-1]{\frac{1-\alpha}{(1-\alpha / k)\left|a_{k}\right|}} \leq 1, \quad\left|a_{k}\right| \neq 0 .
$$

This completes the proof.

Remark 2 For example, the values $\alpha=0.5, k=2$ and $\left|a_{k}\right|=1$ imply the radius of starlikeness of $S_{k}(z)$ is $r=0.8164965 \ldots$, and for the same values, the radius of starlikeness of the ordinary partial sums $f_{k}(z)=z+a_{k} z^{k}$ is $r=0.577350 \ldots$ (see [2]).

Next, we derive the radius of convexity. 
Theorem 3 The function $S_{k}(z)$ satisfies

$$
\frac{1-k\left|a_{k}\right| r^{k-1}}{1-\left|a_{k}\right| r^{k-1}} \leq \Re\left(1+\frac{z S_{k}^{\prime \prime}(z)}{S_{k}^{\prime}(z)}\right) \leq \frac{1+k\left|a_{k}\right| r^{k-1}}{1+\left|a_{k}\right| r^{k-1}}
$$

for

$$
0 \leq r<\sqrt[k-1]{\frac{1}{\left|a_{k}\right|}} \leq 1, \quad\left|a_{k}\right| \neq 0 .
$$

Furthermore, $S_{k}(z) \in \mathcal{C}(\alpha)$ for

$$
0 \leq r<\sqrt[k-1]{\frac{1-\alpha}{(k-\alpha)\left|a_{k}\right|}} \leq 1, \quad\left|a_{k}\right| \neq 0 .
$$

Proof A computation gives

$$
1+\frac{z S_{k}^{\prime \prime}(z)}{S_{k}^{\prime}(z)}=k-\frac{(k-1)}{1+a_{k} z^{k-1}}
$$

Therefore, for $\cos \theta \rightarrow 1$, we obtain

$$
\begin{aligned}
\Re\left(1+\frac{z S_{k}^{\prime \prime}(z)}{S_{k}^{\prime}(z)}\right) & =k-(k-1) \frac{1+\left|a_{k}\right| \cos \theta r^{k-1}}{1+2\left|a_{k}\right| r^{k-1} \cos \theta+\left|a_{k}\right|^{2} r^{2(k-1)}} \\
& \leq \frac{1+k\left|a_{k}\right| r^{k-1}}{1+\left|a_{k}\right| r^{k-1}} .
\end{aligned}
$$

Moreover, we impose

$$
\Re\left(1+\frac{z S_{k}^{\prime \prime}(z)}{S_{k}^{\prime}(z)}\right) \geq \frac{1-k\left|a_{k}\right| r^{k-1}}{1-\left|a_{k}\right| r^{k-1}} .
$$

Now, consider that

$$
\frac{1-k\left|a_{k}\right| r^{k-1}}{1-\left|a_{k}\right| r^{k-1}}>\alpha
$$

for

$$
0 \leq r<\sqrt[k-1]{\frac{1-\alpha}{(k-\alpha)\left|a_{k}\right|}} \leq 1, \quad\left|a_{k}\right| \neq 0 .
$$

This completes the proof.

Remark 4 In view of Theorem 3, for example, the values $\alpha=0.5, k=2$ and $\left|a_{k}\right|=1$ pose the radius of convexity of $S_{k}(z)$ is $r=0.577350 \ldots$ and for the same values, the radius of convexity of the ordinary partial sums $f_{k}(z)=z+a_{k} z^{k}$ is $r=0.4082 \ldots$ (see [2]).

Next, we assume special ordinary partial sums depending so that their coefficients satisfy the relation $\left|a_{n}\right| \leq\left(\frac{k-n+1}{k}\right)$. 
Theorem 5 Assume the partial sum

$$
f_{3}(z)=z+\frac{k-1}{k} z^{2}+\frac{k-2}{k} z^{3}, \quad k \geq 2 .
$$

Then the function $f_{3}(z) \in S^{*}\left(\frac{1}{2}\right)$.

Proof We consider $\alpha$ such that

$$
\Re\left(\frac{z f_{3}^{\prime}(z)}{f_{3}(z)}\right)=\mathfrak{R}\left(3-\frac{2+\frac{k-1}{k} z}{1+\frac{k-1}{k} z+\frac{k-2}{k} z^{2}}\right)>\alpha .
$$

This implies that

$$
\Re\left(\frac{2+\frac{k-1}{k} z}{1+\frac{k-1}{k} z+\frac{k-2}{k} z^{2}}\right)<3-\alpha,
$$

that is,

$$
\Re\left(\frac{1-\frac{k-1}{k} z^{2}}{1+\frac{k-1}{k} z+\frac{k-2}{k} z^{2}}\right)=\frac{1-\frac{k-1}{k} r^{2}\left(2 \cos ^{2} \theta-1\right)}{1+\frac{k-1}{k} r \cos \theta+\frac{k-2}{k} r^{2}\left(2 \cos ^{2} \theta-1\right)}<2-\alpha .
$$

By letting $t=\cos \theta$, we define the function $g(t)$ as follows:

$$
g(t)=\frac{1-\frac{k-1}{k} r^{2}\left(2 t^{2}-1\right)}{1+\frac{k-1}{k} r t+\frac{k-2}{k} r^{2}\left(2 t^{2}-1\right)} .
$$

Logarithmic derivative of $g(t)$ yields

$$
\begin{aligned}
\frac{g^{\prime}(t)}{g(t)} & =-\left\{\frac{\left(4 t \frac{k-1}{k} r^{2}\right)\left[1+\frac{k-1}{k} r t+\frac{k-2}{k} r^{2}\left(2 t^{2}-1\right)\right]+\left(\frac{k-1}{k} r+4 \frac{k-2}{k} r^{2} t\right)\left[1-\frac{k-1}{k} r^{2}\left(2 t^{2}-1\right)\right]}{\left[1-\frac{k-1}{k} r^{2}\left(2 t^{2}-1\right)\right]\left[1+\frac{k-1}{k} r t+\frac{k-2}{k} r^{2}\left(2 t^{2}-1\right)\right]}\right\} \\
& :=-\left\{\frac{h(t)}{\left[1-\frac{k-1}{k} r^{2}\left(2 t^{2}-1\right)\right]\left[1+\frac{k-1}{k} r t+\frac{k-2}{k} r^{2}\left(2 t^{2}-1\right)\right]}\right\} \\
& =-\frac{A t^{2}+B t+C}{\left[1-\frac{k-1}{k} r^{2}\left(2 t^{2}-1\right)\right]\left[1+\frac{k-1}{k} r t+\frac{k-2}{k} r^{2}\left(2 t^{2}-1\right)\right]},
\end{aligned}
$$

where

$$
\begin{aligned}
& A=2 r^{3}\left(\frac{k-1}{k}\right)^{2}, \\
& B=4 r^{2}\left[\frac{k-1}{k}+\frac{k-2}{k}\right], \\
& C=\frac{k-1}{k} r\left[1+\frac{k-1}{k} r^{2}\right] .
\end{aligned}
$$

Now, for all $k \geq 2$ and $r \rightarrow 1$, the function $h(t)$ has unique real negative zeros in the interval $\left(-\frac{1}{2}, 0\right)$. This leads to the fact that $g^{\prime}(t)$ has unique positive real zeros for all $k \geq 2$ 
distributed in the interval $\left(0, \frac{1}{2}\right)$. Therefore, we will calculate $\alpha$ in $t \in\left[\frac{1}{2}, 1\right)$. It is easy to check that $g(t)$ is decreasing for $r \rightarrow 1$ in the interval $\left[\frac{1}{2}, 1\right)$. Moreover, we have

$$
\lim _{k \rightarrow \infty} g\left(\frac{1}{2}\right)=\lim _{k \rightarrow \infty} \frac{1+\frac{k-1}{2 k}}{1+\frac{k-1}{2 k}-\frac{k-2}{2 k}}=\frac{3}{2}
$$

We conclude that for $t \in\left[\frac{1}{2}, 1\right)$,

$$
g(t)<g\left(\frac{1}{2}\right) \leq \frac{3}{2}=2-\alpha
$$

thus $\alpha=\frac{1}{2}$. This completes the proof.

By letting $k=3$ in Theorem 5 , we have the following result.

\section{Corollary 6 The Cesáro partial sums}

$$
\sigma_{3}(z)=z+\frac{2}{3} z^{2}+\frac{1}{3} z^{3}, \quad z \in U
$$

of the function $f(z)=\frac{z}{1-z}$ are starlike of order $\alpha=\frac{1}{5}$.

Theorem 7 Assume the partial $\operatorname{sum}_{3}(z)$ as in Theorem 5. Then the function $f_{3}(z) \in \mathcal{C}\left(\frac{1}{5}\right)$.

Proof We consider $\alpha$ such that

$$
\Re\left(1+\frac{z f_{3}^{\prime \prime}(z)}{f_{3}^{\prime}(z)}\right)=\Re\left(3-\frac{2\left(\frac{k-1}{k} z+1\right)}{1+2 \frac{k-1}{k} z+3 \frac{k-2}{k} z^{2}}\right)>\alpha .
$$

This implies that

$$
\Re\left(\frac{\frac{k-1}{k} z+1}{1+2 \frac{k-1}{k} z+3 \frac{k-2}{k} z^{2}}\right)<\frac{3-\alpha}{2},
$$

therefore, a computation gives

$$
\Re\left(\frac{\frac{k-1}{k} z+1}{1+2 \frac{k-1}{k} z+3 \frac{k-2}{k} z^{2}}\right)=\frac{1}{2}+\Re\left(\frac{\frac{1}{2}\left(1-3 \frac{k-2}{k} z^{2}\right)}{1+2\left(\frac{k-1}{k}\right) z+3\left(\frac{k-2}{k}\right) z^{2}}\right),
$$

thus

$$
\frac{\frac{1}{2}\left(1-3 \frac{k-2}{k} r^{2}\left(2 \cos ^{2} \theta-1\right)\right)}{1+2\left(\frac{k-1}{k}\right) r \cos \theta+3\left(\frac{k-2}{k}\right)\left(2 \cos ^{2} \theta-1\right)}<\frac{2-\alpha}{2} .
$$

By putting $t=\cos \theta$, we define the function $G(t)$ as follows:

$$
G(t)=\frac{\frac{1}{2}\left(1-3 \frac{k-2}{k} r^{2}\left(2 t^{2}-1\right)\right)}{1+2\left(\frac{k-1}{k}\right) r t+3 r^{2}\left(\frac{k-2}{k}\right)\left(2 t^{2}-1\right)} .
$$


Logarithmic derivative of $G(t)$ yields

$$
\begin{aligned}
\frac{G^{\prime}(t)}{G(t)}= & -\left\{\frac{\left[12 r^{2} \frac{k-2}{k} t\right]\left[1+2\left(\frac{k-1}{k}\right) r t+3 r^{2}\left(\frac{k-2}{k}\right)\left(2 t^{2}-1\right)\right]}{\left[1-3 \frac{k-2}{k} r^{2}\left(2 t^{2}-1\right)\right]\left[1+2\left(\frac{k-1}{k}\right) r t+3 r^{2}\left(\frac{k-2}{k}\right)\left(2 t^{2}-1\right)\right]}\right. \\
& \left.+\frac{\left[12 r^{2} \frac{k-2}{k} t+2 r \frac{k-1}{k}\right]\left[1-3 \frac{k-2}{k} r^{2}\left(2 t^{2}-1\right)\right]}{\left[1-3 \frac{k-2}{k} r^{2}\left(2 t^{2}-1\right)\right]\left[1+2\left(\frac{k-1}{k}\right) r t+3 r^{2}\left(\frac{k-2}{k}\right)\left(2 t^{2}-1\right)\right]}\right\} \\
:= & -\left\{\frac{H(t)}{\left[1-3 \frac{k-2}{k} r^{2}\left(2 t^{2}-1\right)\right]\left[1+2\left(\frac{k-1}{k}\right) r t+3 r^{2}\left(\frac{k-2}{k}\right)\left(2 t^{2}-1\right)\right]}\right\} \\
= & -\frac{A t^{2}+B t+C}{\left[1-3 \frac{k-2}{k} r^{2}\left(2 t^{2}-1\right)\right]\left[1+2\left(\frac{k-1}{k}\right) r t+3 r^{2}\left(\frac{k-2}{k}\right)\left(2 t^{2}-1\right)\right]},
\end{aligned}
$$

where

$$
\begin{aligned}
& A=12 r^{3} \frac{(k-1)(k-2)}{k^{2}}, \\
& B=12 r^{2} \frac{k-2}{k}\left[2+3 r^{2}\left(\frac{k-2}{k}\right)\right], \\
& C=2 r \frac{k-1}{k}\left[1+3 r^{2}\left(\frac{k-2}{k}\right)\right] .
\end{aligned}
$$

Now, for all $k \geq 3$ and $r \rightarrow 1$, the function $H(t)$ has unique real negative zeros in the interval $\left[-\frac{1}{2}, 0\right)$. This leads to the fact that $G^{\prime}(t)$ has unique positive real zeros for all $k \geq 3$ in the interval $\left(0, \frac{1}{2}\right]$. So, we calculate $\alpha$ in the interval $t \in\left(\frac{1}{2}, 1\right)$. A computation yields $G(t)$ is decreasing for $r \rightarrow 1$ in the interval $t \in\left(\frac{1}{2}, 1\right)$. Thus, we have

$$
\lim _{k \rightarrow \infty} G(t)<\frac{9}{10}=\frac{2-\alpha}{2}, \quad 1>t>0.5
$$

which implies $\alpha=\frac{1}{5}$. This completes the proof.

Theorem 8 Assume the Cesáro partial sum

$$
\sigma_{3}(z)=z+\frac{4}{3} z^{2}+z^{3}
$$

of the function

$$
f(z)=\frac{z}{(1-z)^{2}}=z+2 z^{2}+3 z^{3}+\cdots .
$$

Then the function $\sigma_{3}(z) \in \mathcal{C}\left(\frac{1}{2}\right)$ for all $0.2<r<0.5$.

Proof We consider $\alpha$ such that

$$
\Re\left(1+\frac{z \sigma_{3}^{\prime \prime}(z)}{\sigma_{3}^{\prime}(z)}\right)=\Re\left(3-\frac{2\left(\frac{4}{3} z+1\right)}{1+\frac{8}{3} z+3 z^{2}}\right)>\alpha .
$$

This implies that

$$
\Re\left(\frac{\frac{4}{3} z+1}{1+\frac{8}{3} z+3 z^{2}}\right)<\frac{3-\alpha}{2},
$$


therefore, a computation gives

$$
\Re\left(\frac{\frac{4}{3} z+1}{1+\frac{8}{3} z+3 z^{2}}\right)=\frac{1}{2}+\Re\left(\frac{\frac{1}{2}\left(1-3 z^{2}\right)}{1+\frac{8}{3} z+3 z^{2}}\right),
$$

thus

$$
\frac{1-3 r^{2}\left(2 \cos ^{2} \theta-1\right)}{1+\frac{8}{3} r \cos \theta+3\left(2 \cos ^{2} \theta-1\right)}<2-\alpha .
$$

By putting $t=\cos \theta$, we define the function $J(t)$ as follows:

$$
J(t)=\frac{1-3 r^{2}\left(2 t^{2}-1\right)}{1+\frac{8}{3} r t+3 r^{2}\left(2 t^{2}-1\right)} .
$$

Logarithmic derivative of $J(t)$ yields

$$
\begin{aligned}
\frac{J^{\prime}(t)}{J(t)} & :=-\left\{\frac{\hbar(t)}{\left[1-3 r^{2}\left(2 t^{2}-1\right)\right]\left[1+\frac{8}{3} r t+3 r^{2}\left(2 t^{2}-1\right)\right]}\right\} \\
& =-\frac{16 r^{3} t^{2}+24 r^{2} t+\frac{8}{3} r\left(1+3 r^{2}\right)}{\left[1-3 r^{2}\left(2 t^{2}-1\right)\right]\left[1+\frac{8}{3} r t+3 r^{2}\left(2 t^{2}-1\right)\right]}
\end{aligned}
$$

The function $\hbar(t)$ has a unique real negative zero in the interval $t \in(-1,0)$ for all $0.2<r<$ 0.5 which is around $t \simeq-\frac{1}{2}$. This leads to the fact that $J^{\prime}(t)$ has a unique positive real zero in the interval $(0,1)$ around $t \simeq \frac{1}{2}$. A computation yields $J(t)$ is decreasing in the interval $t \in\left(\frac{1}{2}, 1\right)$ and assuming its maximums at $t=0.5$ and $r=0.5$. Thus, we have

$$
\lim _{r \rightarrow 0.5, t \rightarrow 0.5} J(t)<\frac{3}{2}=2-\alpha,
$$

which implies $\alpha=\frac{1}{2}$. This completes the proof.

Note that some other results related to partial sums can be found in [11-15].

\section{Competing interests}

The authors declare that they have no competing interests.

\section{Authors' contributions}

Both authors jointly worked on deriving the results and approved the final manuscript.

\section{Author details}

${ }^{1}$ Institute of Mathematical Sciences, Universiti Malaya, Kuala Lumpur, 50603, Malaysia. ${ }^{2}$ School of Mathematical Sciences, Faculty of Science and Technology, Universiti Kebangsaan Malaysia, Bangi, Selangor Darul Ehsan 43600, Malaysia.

\section{Acknowledgements}

The work was fully supported by UKM-DLP-2011-050 and LRGS/TD/2011/UKM/ICT/03/02. The authors also would like to thank the referees for giving some suggestions for improving the work.

Received: 4 August 2012 Accepted: 25 January 2013 Published: 14 February 2013

\section{References}

1. Szego, G: Zur theorie der schlichten abbilungen. Math. Ann. 100, 188-211 (1928)

2. Owa, S: Partial sums of certain analytic functions. Int. J. Math. Math. Sci. 25(12), 771-775 (2001)

3. Darus, M, Ibrahim, RW: Partial sums of analytic functions of bounded turning with applications. Comput. Appl. Math. 29(1), 81-88 (2010) 
4. Robertson, MS: On the univalency of Cesáro sums of univalent functions. Bull. Am. Math. Soc. 42, 241-243 (1936)

5. Ruscheweyha, S, Salinas, LC: Subordination by Cesáro means. Complex Var. Elliptic Equ. 3(21), 279-285 (1993)

6. Splina, LT: On certain applications of the Hadamard product. Appl. Math. Comput. 199, 653-662 (2008)

7. Darus, M, Ibrahim, RW: On Cesáro means for Fox-Wright functions. J. Math. Stat. 4(3), 156-160 (2008)

8. Darus, M, Ibrahim, RW: On some properties of differential operator. Acta Didact. Napoc. 2(2), 1-6 (2009)

9. Darus, M, Ibrahim, RW: Coefficient inequalities for concave Cesáro operator of non-concave analytic functions. Eur. J. Pure Appl. Math. 3(6), 1086-1092 (2010)

10. Srivastava, HM, Darus, M, Ibrahim, RW: Classes of analytic functions with fractional powers defined by means of a certain linear operator. Integral Transforms Spec. Funct. 1(22), 17-28 (2011)

11. Frasin, BA: Generalization of partial sums of certain analytic and univalent functions. Appl. Math. Lett. 21, 735-741 (2008)

12. Wang, Z-G, Liu, Z-H, Catas, A: On neighborhoods and partial sums of certain meromorphic multivalent functions. Appl. Math. Lett. 24, 864-868 (2011)

13. Murugusundaramoorthy, G, Uma, K, Darus, M: Partial sums of generalized class of analytic functions involving Hurwitz-Lerch zeta function. Abstr. Appl. Anal. 2011. Article ID 849250 (2011). doi:10.1155/2011/849250

14. Ghanim, F, Darus, M: Partial sums of certain new subclasses for meromorphic functions. Far East J. Math. Sci. 55(2), 181-195 (2011)

15. Ibrahim, RW, Darus, M: Partial sums for certain classes of meromorphic functions. Tamkang J. Math. 41(1), 39-49 (2010)

doi:10.1186/1029-242X-2013-51

Cite this article as: Ibrahim and Darus: Cesáro partial sums of certain analytic functions. Journal of Inequalities and Applications 2013 2013:51.

\section{Submit your manuscript to a SpringerOpen ${ }^{\circ}$ journal and benefit from:}

- Convenient online submission

- Rigorous peer review

- Immediate publication on acceptance

- Open access: articles freely available online

- High visibility within the field

- Retaining the copyright to your article 